\title{
Lip and perioral assessment scale - a tool that can improve your practice
}

\begin{abstract}
Background: The lips and perioral region are a highly dynamic anatomic area of the face and its anatomy and measurements will vary from patient to patient. In many years of clinical practice and as trainers for other injectors we have noticed that the assessment of this region is also very variable regardless of the experience of the injector.

Objectives: The goal of this article is to provide a tool for assessment of the lower face and perioral area in other to simplify cosmetic treatments and help injectors identify what needs to be addressed in each patient.

Methods: Over the last few years, weanalyzed the most common concerns of every patient presenting for treatment of the lips and perioral area and the most frequent areas addressed in the treatment of the lower face, and organized them into a tool that was able to systematize the different levels of attention needed in each particular area, guiding injectors towards a better final result. As trainers for other injectors, we also analyzed the most difficult aspects of the perioral assessment and simplified them into this tool that can help even novice injectors to do the correct diagnosis and therefore achieve better results while treating the perioral area.

Conclusions: We believe that this tool will help both experienced and young injectors in the assessment of the lips and perioral area prior to treatment and in doing so, will provide a better outcome and cosmetic result.
\end{abstract}

Keywords: lip fillers, perioral treatments, hyaluronic acid, perioral assessment, facial assessment
Volume 5 Issue 4 - 202I

\author{
Luiz Eduardo Toledo Avelar, Alessandra \\ Haddad, Juliana Horbilon, Lorena Miranda \\ Avelar, Julia Real \\ Department of plastic surgery, Faculdade Ciências Médicas de \\ Minas Gerais, Brazil
}

Correspondence: Luiz Eduardo Toledo Avelar, Department of plastic surgery, Faculdade Ciências Médicas de Minas Gerais, Brazil,Email contato@luizeduardoavelar.com.br

Received: August 16,202I | Published: September 27, 2021

\section{Introduction}

Lips are considered one of the most important features of facial aesthetics due to their central position in the face and their elemental role in verbal and nonverbal interaction, being able to communicate emotion, even while the face is at rest. ${ }^{1-13}$ Lip shape, contour, fullness and projection play a major part in overall composition of facial aesthetics and outward appearance and are intrinsically linked to selfperception of attractiveness, self-esteem, and social confidence..$^{5,6}$ The importance of the lip in overall facial aesthetics has an impact on positive self-image and self-confidence. ${ }^{6}$

In virtually all women, since the beginning of recorded history, full lips have been associated with youth and beauty ${ }^{3,5,7,13}$ and therefore, lip enhancement is one of the most frequently requested procedures in a cosmetic practice nowadays. . $^{3,5,6}$

The injection of dermal fillers is a minimally invasive procedure that has become increasingly popular worldwide and is considered the most popular nonsurgical procedure performed to increase the volume and improve the shape of the lips. $3,5,6,7,9$

Lips become thinner with age due to a decrease in collagen and elastin, but only filling the vermillion with hyaluronic acid most likely will not give our patients an excellent result.

Clinically, perioral aging is an increasingly frequent patient concern and one that has proven to be excessively difficult to treat in a natural manner. ${ }^{8}$ A comprehensive understanding of the anatomical features of the perioral area and the aging process is foundational information for an injector. Whether the goal is to enhance youthful lips or restore shape and volume to aging lips, an understanding of the relevant anatomy and the ability to employ a suitable corrective need is very important. ${ }^{6}$ As the aging process is multifactorial, lip rejuvenation has several aims other than just restoring volume, including diminishing fine lines, redefining the cupid's bow and vermilion border, shortening the length of the cutaneous upper lip and eversion of the vermilion.

Age-related changes in all anatomical layers of the face, including the underlying skeleton, soft tissues such as fat compartments and muscles, as well as the skin, interdependently contribute to the phenotype of the aging face. ${ }^{8}$ With gradual loss of dental and bone support, it is common to see a collapse of the upper lip projection with atrophy and development of a labial entropion, a lengthening of the upper lip and an alteration of the proportions of white upper lip, vermillion and red upper lip. The later decreases significantly with aging. The vermillion border can lose definition and the once prominent tubercles, cupid's bow and philtrum columns can become blunted. ${ }^{5,6}$ Genetics, intrinsic aging, sun exposure, smoking, and repetitive pursing of the orbicularis oris muscle produce angular, radial, and vertical "lipstick bleed lines", that are very common, specially in women. ${ }^{5}$ Aging also leads to pallor of the vermilion that result in the loss of sharp vermilion-cutaneous junction demarcation. ${ }^{5,6}$

Perioral rejuvenation remains one of the most challenging, complex, and controversial aspects of aesthetic surgery. ${ }^{8}$ In this article, we will address only aspects of facial assessment and how it can help guide the treatment with fillers of the perioral area despite patient age.

\section{Methods}

We analyzed all patients who presented in our offices with the desire to improve the lips and the perioral area between January 2018 
and January 2021. We treated 980 patients ageing 18 to 78 years of age; 108 were male and 872 were female. All patients were photographed at the time of the treatment with hyaluronic acid fillers, 30 days and 90 days after the treatment. These photos were analyzed and discussed by each of the authors regarding patient concern, perioral assessment of each author and the type of treatment and techniques performed at the time.

By systematizing this analysis we could come up with this tool that can guide injectors towards an easier assessment of the perioral region, improve the time spent planning the treatments and provide better results as the treatments become more assertive, regardless of the experience of the injector.

\section{First step - perioral evaluation}

Many patients will present with the desire of getting their lips enhanced, but both patient and physician often ignore the perioral area, that is just as important. The lips are the most prominent part of the perioral area but if the area is depressed due to aging, the lips may lose their structural platform. The corrective needs of a patient, regardless of age, can range from a subtle enhancement to a complete recontouring, of the perioral area. ${ }^{6}$ When analyzing the lips for cosmetic treatment, it is mandatory that we also consider the perioral area, including the piriform aperture, nasolabial folds, marionette lines and the chin.
When seeing a patient for the first time, it is mandatory that we evaluate he bone structure, dental occlusion and surrounding tissues in order to give them an excellent result, and not only fuller lips.

When we evaluate the perioral area, we must have all structures in mind, from deep to superficial: bone, muscle, subcutaneous tissues and skin. As we start to plan treatments for the lips, we should begin by analyzing all structures around the perioral area. The bone structure and position of the mandible, maxilla and teeth placement interfere in the projection of each lip.

Edentulous patients, for example, will most likely lack lip projection. Therefore, it is important to assess dental occlusion and chin projection as part of the evaluation of the underling bone structure of our patient. Often times, patients with Angle's Class II malocclusion will lack projection of the lower lip and Class III malocclusion patients will lack projection of the upper lip.

We present a 47 year old patient (Figure 1,2) that lacks chin and lower lip projection due to her bone structure. We treated her entire lower face and surrounding tissues of the perioral area, giving her more structure and projection of the midface / medial malar area, more chin projection, myomodulated the mentalis and orbicularis oris muscles (treating the bar code lines of the upper lip as well) and filled the nasolabial folds and marionette lines, using a total of $4 \mathrm{ml}$ of hyaluronic acid.

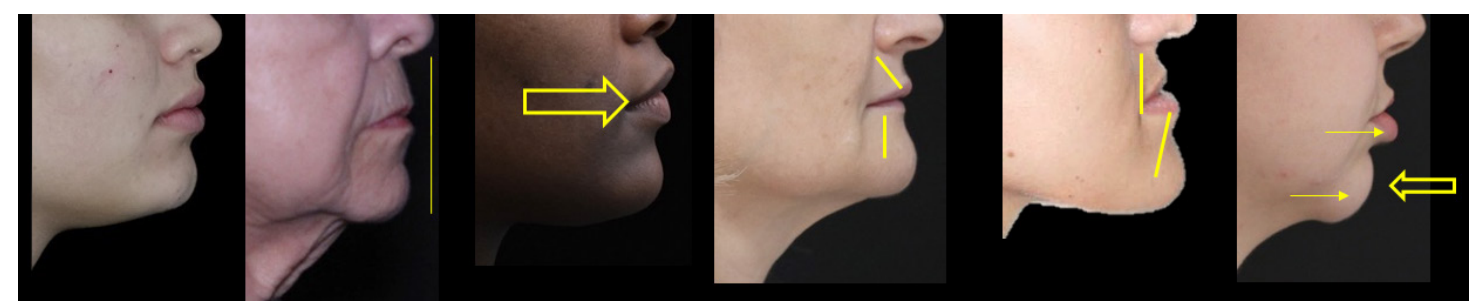

Figure I Relationship between underling structures and lip projection in different patients ages 18-62.

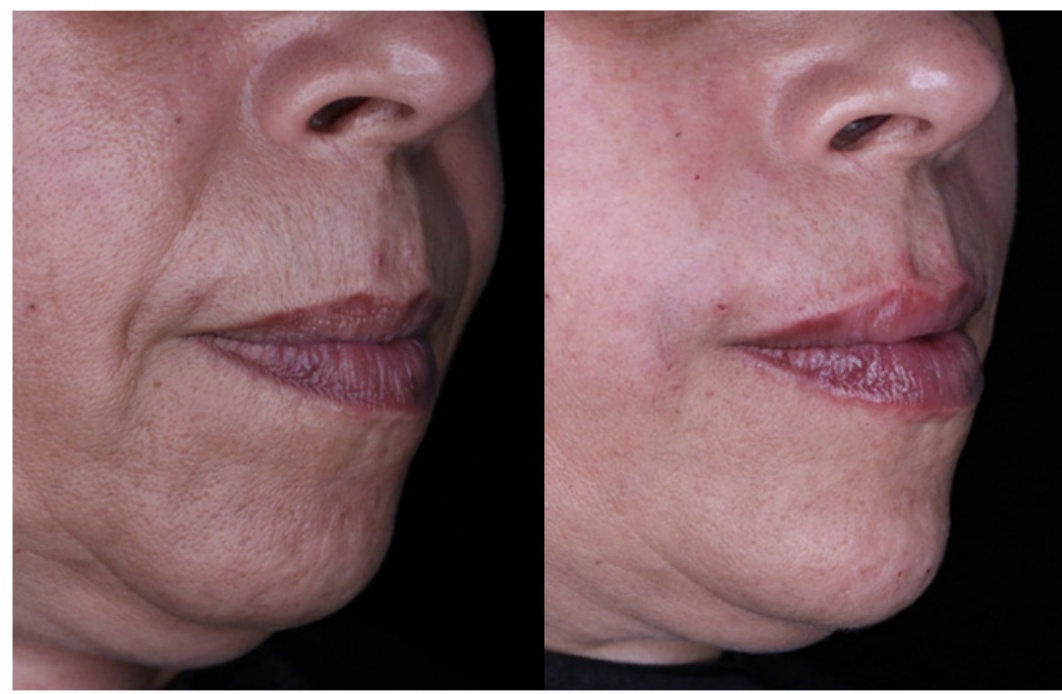

Figure 2 Perioral and chin correction influencing the shape of the lips - immediate result, 47 year old parient.

After assessing the bone structure of the lower face, we can move on to examining the muscles of the perioral area. Because the lip and perioral region experience regular dynamic movement, adding filler to enhance shape while still maintaining a natural appearance (during movement and at rest) can be a challenge to many injectors. ${ }^{6,8}$
Numerous muscles are located around the lips and allow for a wide range of facial expressions and functions such as speaking, eating and kissing. The orbicularis oris muscle has a superficial portion inserting fibers on the skin of the lip area that can lead to bar code lines as we age. 
The modiolus angulioris is a chiasma of facial muscles held together by fibrous tissue, located lateral and slightly superior to each angle of the mouth. ${ }^{12}$ Several muscles can be part the modiolus: orbicularis oris, levator anguli oris, zygomaticus major, buccinator, risorius, depressor angulioris (DAO) and platysma pars modiolaris.

The DAO is a muscle stronger than the others, and because it is continuing pulling the corners of the mouth downward, with age, it gives the patients the appearance of sadness. Combined with gravity, osteoporosis, dental changes, maxillomandibular bony resorption, and further soft tissue volume loss at the oral commissures it causes the commissures to turn downward in a perpetual frown. This is worsened by a combination of midfacial aging with ligamentous laxity in the cheeks, causing the formation of jowls and "marionette" lines that extend downward from the oral commissures to the mandible (Figure 3-5).

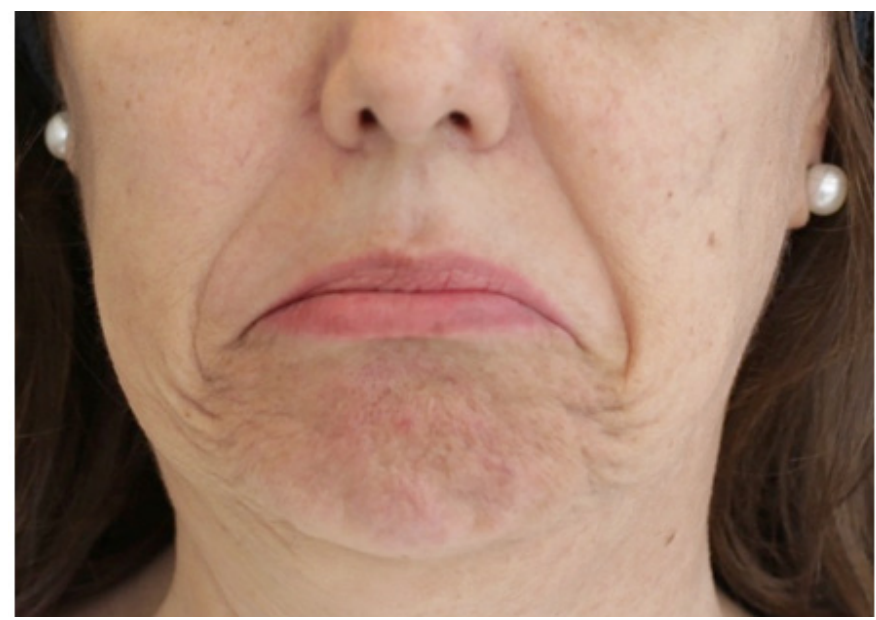

Figure 350 year old female patient contracting the DAO muscles.
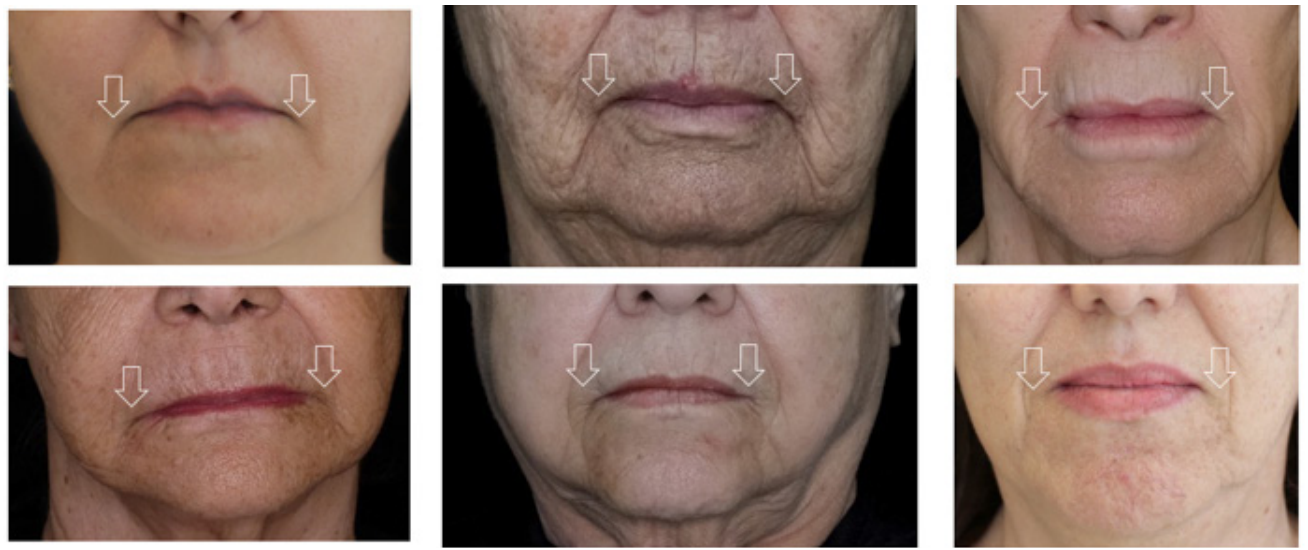

Figure 4 Series of patients ages $45-70$ in rest position but with the corners of the mouth pointing downwards.

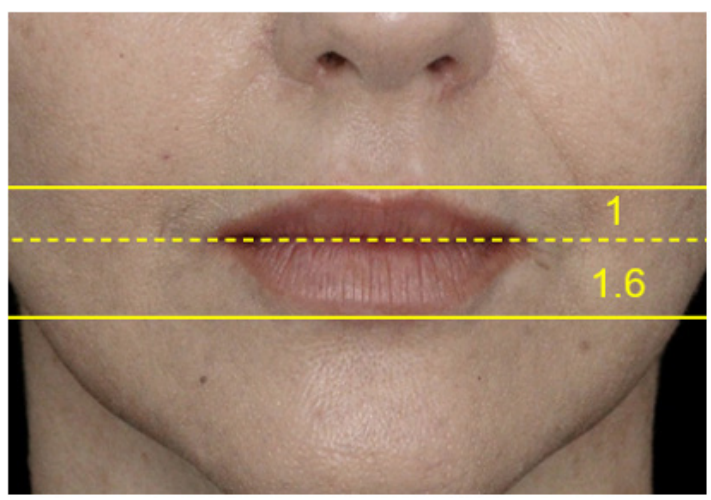

Figure 5 The ideal ratio of lip height I:I,6.

The downward pointing corner of the mouth can be treated by undermining the area with a cannula before injecting hyaluronic acid. This will help free the skin of the DAO fibers and the hyaluronic acid superficially will act as way to myomodulate the DAO muscle. Injectable neuromodulating agents (botulinum toxin A) placed into the depressor anguli oris muscles can be used as an adjunct to further elevate the commissures. This combines structural support with chemical neuromodulation to shape the oral commissures. ${ }^{5}$

The labiomental crease passes horizontally in an inverted U-shape across the lower lip, which intraorally corresponds to the depth of the gingivolabial sulcus. ${ }^{12}$ The labiomental crease can become more prominent as we age and can be treated by undermining the skin with a cannula and subsequent placement of filler.After treating the surrounding tissues, we can start evaluating the lips, their projection and proportions.

\section{Second step- lips evaluation}

The lips are one of the most important elements of the human face, being a central feature of the face and having a key role in expressing emotions and social interactions such as speaking, kissing and eating. 
Therefore, the lips are a major patient concern regarding aesthetic treatments for both young and older patients seeking either better form, volume or symmetry or restauration of what was lost due to the aging process.

The upper lip is delimited upwards by the nose, downwards by the mouth horizontal axis, and laterally by the nasolabial folds. ${ }^{2}$ The upper lip has anatomical sub-units that need to be known and preserved when treating the lips with hyaluronic acid fillers.

- The philtral columns are the two ridges on the central upper lip - they can be very well defined in some patients or somewhat blurred in others.

- The philtral columns form a midline depression called the philtrum and are responsible for the formation of the Cupid 's bow. ${ }^{12}$

- The Cupid's bow is the convex segment of the vermillion border located directly under the philtrum. The philtrum extends from the vermilion superiorly to the columella in an inverted V-shape.

The edge where the red colored vermillion body meets the surrounding skin is known as the vermillion border. This edge also has a slight elevation, known as the white roll. ${ }^{6}$

The outer layer of the white lip consists of skin containing glands and hair follicles and the outer layer of the vermillion is a thin mucosa with no glands, therefore, being prone to dehydration. The middle layer consists of the orbicularis oris muscle and the inner layer is a wet mucosal membrane that contains numerous sebaceous glands.

The vermillion is composed of numerous capillaries, which give its characteristic pink/red color. ${ }^{12}$ The lip and perioral region are also densely vascularized and vulnerable to injection site reactions, such as edema, bruising and vascular occlusion. ${ }^{6}$

Frequent application of volumizing materials in the head and neck region has led to multiple serious complications, including vascular compromise or occlusion leading to cutaneous necrosis and blindness. Therefore, injectors require an in-depth understanding of the anatomy and vasculature of the lips and the perioral area prior to providing any treatment using dermal fillers. ${ }^{1,9,10,12,13}$

The facial artery is formed by the branch of the external carotid artery that passes in front of the masseter muscle and behind the depressor anguli oris muscle, through a palpable notch in the mandible. ${ }^{12,13}$ Significant anatomical variation in the course of the facial artery has been demonstrated by several cadaveric studies. The facial artery gives off perioral branches near the commissures with the superior labial artery and inferior labial artery, showing a high variability in presence and course. ${ }^{1,9,10,12,13}$

Cotofana et al.2020 analyzed the location on the labial arteries guided by ultrasound in vivo and found a high number of anatomical variations. The study revealed that the most frequent location of both the superior and the inferior labial arteries is the submucosal plane with $58.5 \%$ followed by the intramuscular $36.2 \%$ and the subcutaneous position $5.3 \%$. The depth of the superior labial artery in the upper lip was $5.6 \pm 1.3 \mathrm{~mm}$ whereas the depth of the inferior labial artery in the lower lip was $5.2 \pm 1.4 \mathrm{~mm}$. The artery is more frequently located within the red lip as opposed to the cutaneous lip: upper lip (83\% vs. $18.7 \%)$ and lower lip (86.2\% vs. $13.8 \%)$; this relationship was more evident especially in the midline, as in all investigated volunteers $(100 \%)$ the artery coursed purely within the red lip. Clinically, these results support an injection technique that is more superficial in the subcutaneous plane independent of labial position. A perpendicular approach to the lip might be safer as the artery courses more frequently within the red lip but it is important to exercise caution regardless of the location of the SLA/ILA because there are always variations. ${ }^{1,9}$

With aging fat pad and muscular changes, the lips become thinner, and the risk of intravascular injection hence increases; more cautious assessment and injections even in the subcutaneous plane, are therefore recommended. ${ }^{12}$

Aesthetic practitioners using dermal fillers must have the competency to diagnose potential complications promptly and be prepared to manage possible immediate vascular complications. It is recommended to always have hyaluronidase available to ensure immediate emergency care if necessary. ${ }^{12}$

Enhancement of lip volume, reformation of the Cupid's bow, and restoration of the vermillion border are frequent indications for the application of materials into the upper and lower lips. ${ }^{9}$ Patients will often present requesting augmentation of the upper or lower lip alone, without considering the balance between the upper and lower lips. It is incumbent upon the physician to educate the patient regarding the ideal ratio between the lips. The physician must establish appropriate guidelines and patient expectations for augmentation relative to normal lip proportions in order to avoid a bizarre appearance. ${ }^{5}$

Although symmetry, fullness, and well demarcated vermillion borders are timeless features of the aesthetic lip, the definition of the ideal lip size, shape and proportions will vary according to patient ethnicity and cultural background of both patient and physician. However, several studies provide a handset of morphological criteria defining what the majority of subjects recognized as a good-looking or attractive face in Caucasian populations. Voluminous dry red lips are perceived as more attractive in several studies and a short upper white lip is highlighted as a criterion of beauty and youth. ${ }^{2}$

On frontal view, an ideal height ratio of the midline upper to lower vermillion in white individuals has been previously defined as 1:1.6 based on the golden ratio. ${ }^{2,4,5,6}$ This can vary with patient desire and ethnicity and some studies reported that this ideal ratio can range between $1: 1$ and 1:1.6 with a ratio closer to $1: 1$ being more suitable for individuals of African ethnicity. 2,6

Recent studies have demonstrated that determining the ideal lip proportions should not only focus on the upper-to-lower lip ratio, but should also include the surface area (SA) and the ratio of the lips to the lower facial third. Using this approach, the lip proportions are not quantified as an absolute ratio, but tailored to specific individual facial proportions. ${ }^{2,6}$

This begins with DaVinci's classic proportions of the lips relative to the rest of the face. ${ }^{5}$ These basic artistic principles from hundreds of years ago still apply. The face is divided vertically into thirds: upper third, middle third and lower third of the face, that in young females should be all equal in vertical height. ${ }^{5,6}$ The lower third of the face is also divided vertically into thirds. Facial proportions vary widely among individuals but according to aesthetic standards, the entire upper lip should represent $1 / 3$ of the inferior third of the face, while the entire lower lip (down to the chin) should represent the remaining 2/3. Those standards apply to both male and female. ${ }^{2,5,6}$ These proportions are altered with aging but not only related to it. Young patients can have altered proportions in need of treatment in order to achieve a well-balanced look of the lower face. 
Anterior projection is also an important element. On the lateral view, the projection of the lips can be evaluated by extending a line from the subnasion point, the inside point of the angle between the septum of the nose and upper lip, to the pogonion, the most anterior mid-point of the chin. The upper lip should project $3.5 \mathrm{~mm}$ and the lower lip should project $2.2 \mathrm{~mm}$ anterior to this line. ${ }^{4,5,6}$

We also consider the Ricketts line for accessing the lip projection, as the lower lip should touch the line or be up to $2 \mathrm{~mm}$ behind it and the superior lip should be $4 \mathrm{~mm}$ behind the line.

\section{Third step- treatment planning scale}

In order to improve the assessment of the lips and the perioral area, we created a scale with three major criteria to be assessed: SURROUNDING TISSUES, PROPORTIONS and LIPS.

The scale may help injectors identify and prioritize where treatment is most needed for a well-balanced and improved result.

For the lips, we will evaluate contour, volume and projection. For the surrounding tissues, we will take into consideration the skin quality, symmetry and dynamic evaluation. For proportions, we will evaluate the lips to the lower face proportions and the superior to the inferior lips proportion.

The goal of a lip augmentation procedure is to create smooth lips with adequate volume and a natural appearance. Various techniques for lip augmentation have been described using a needle or a microcannula or both. The authors present several patients treated with hyaluronic acid fillers and they were treated with various techniques depending on their anatomy and outcome intended.

We will give some examples of how to use the scale to help treatment planning and improving aesthetic results.

As we start to assess this patient, we notice she is a young patient with good skin quality (grade 0 ), a little asymmetric (grade 1) no problems in the dynamic evaluation(grade 0), upper to lower vermillion proportion slightly altered (around 1:1) (grade 1) but a great disproportion between the upper lip and the lower face, (grade3) with a noticeably short upper lip. This points to prioritizing the correction of these proportions, with the need of elongation of the cutaneous upper lip, so we injected her with hyaluronic acid on the anterior nasal spine of the maxilla and on each piriform aperture.

For this 50-year-old patient, (Figure 6-13) the Lip and Perioral Assessment Scale shows some volume loss on both lips due to the aging process. She also has a disproportion between the upper and lower vermillion, with the upper vermillion slightly bigger than the lower. Based on the Scale, we added volume to her lips using $1 \mathrm{ml}$ of hyaluronic acid, but placing more filler in the lower vermillion $(0,7 \mathrm{ml})$ than on the upper $(0,3 \mathrm{ml})$, in order to reinstate the correct proportion between them.
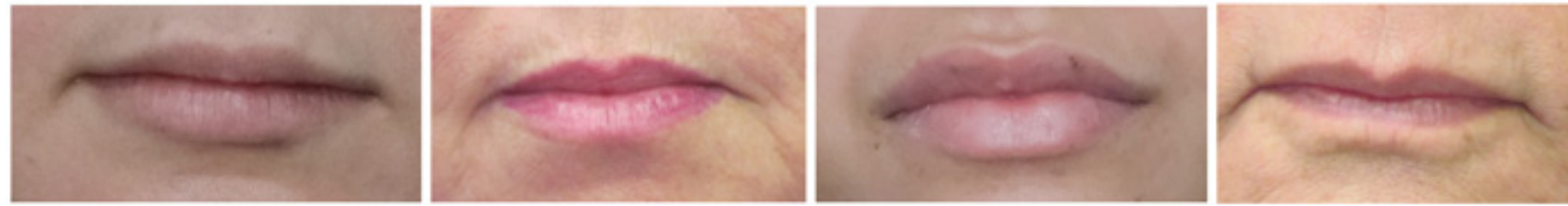

Figure 6 Patients ages 18 to 75 with different lip height proportions.

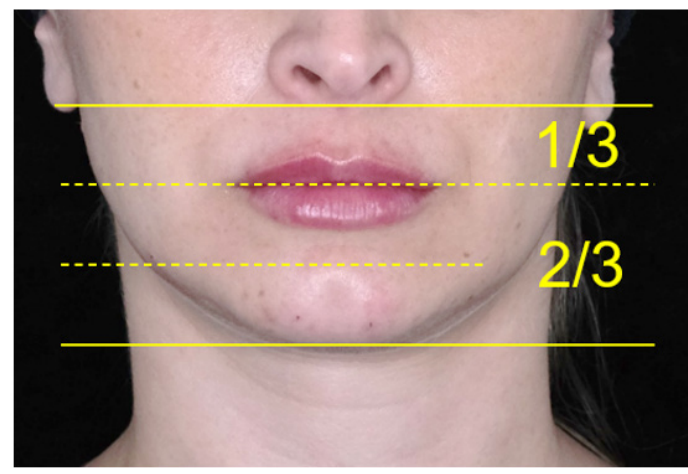

Figure 7 The height of the lips in relation to the lower third of the face.

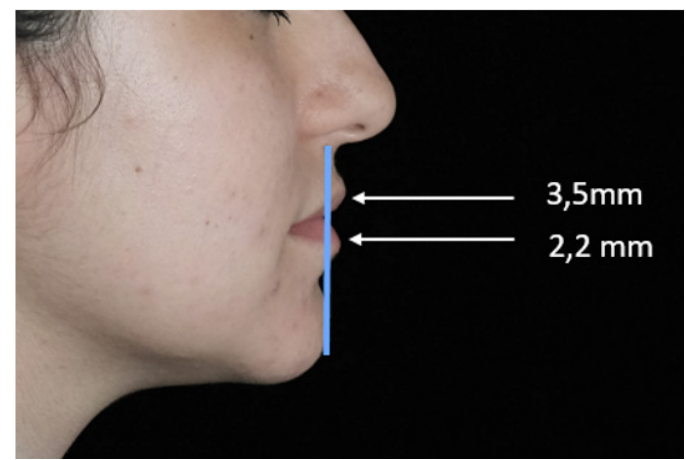

Figure 8 Ideal anterior projection of the upper and lower lips.

Citation: Avelar LET, Haddad A, Horbilon J, et al. Lip and perioral assessment scale - a tool that can improve your practice.J Dermat Cosmetol. 202I;5(4):10I-107. DOI: I0.15406/jdc.202I.05.00191 


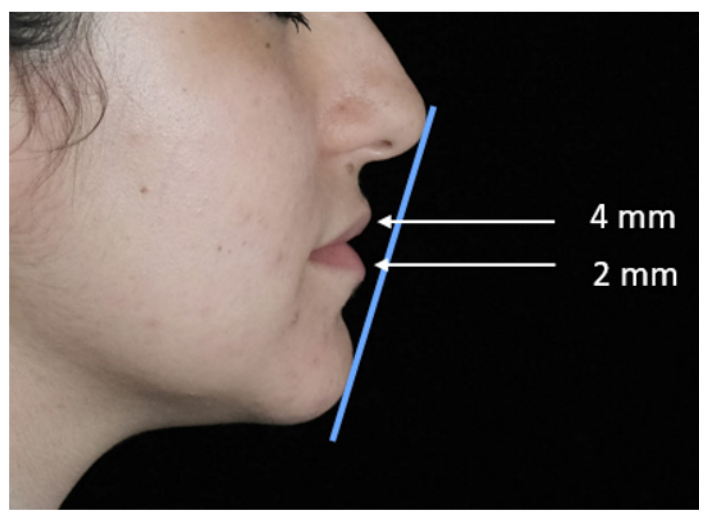

Figure 9 Ricketts line for evaluating the anterior projection of the upper and lower lips.

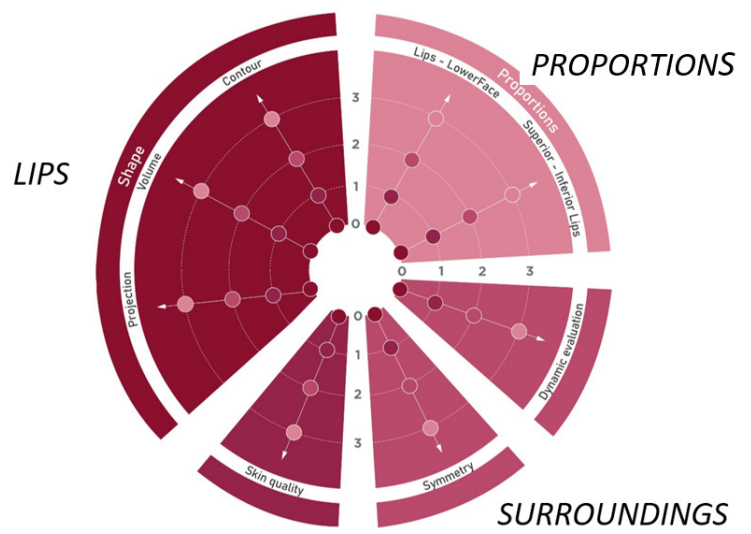

Figure 10 Lip and Perioral Assessment Scale.

Scale degrees from 0 to 3:

0 : no problem in the area

I: small concern in the area

2: moderate concern in the area

3: great concern in the area.

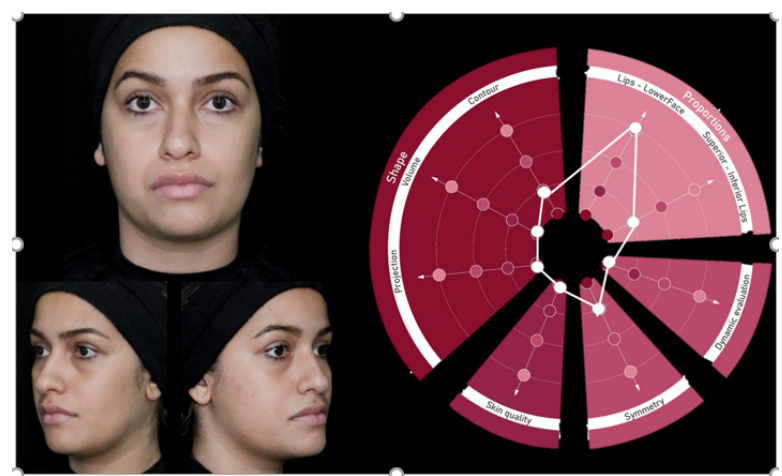

Figure I I Practical example of the Lips Assessment Scale use in a 20-yearold patient.

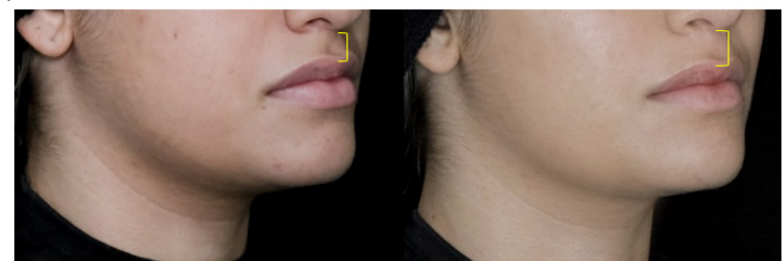

Figure 12 Before and after treatment based on the Lip Assessment Scale of a 20-year-old patient. Note that the lips were not treated.

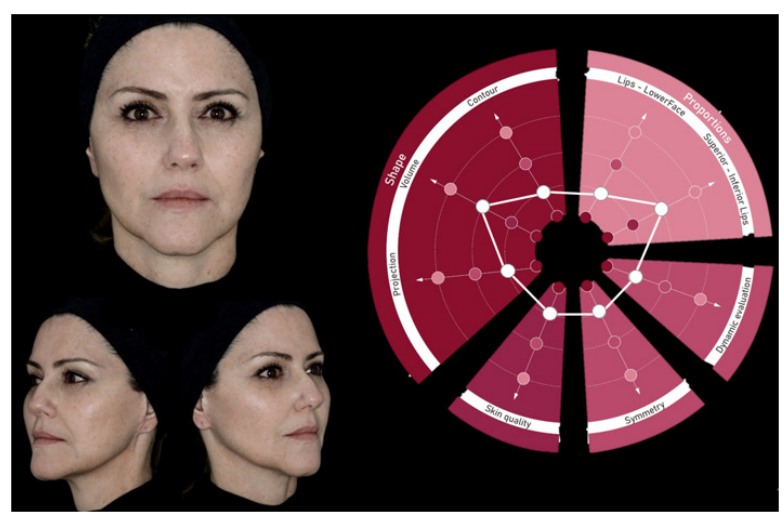

Figure I 3 Practical example of the Lips Assessment Scale use in a 50-yearold patient.

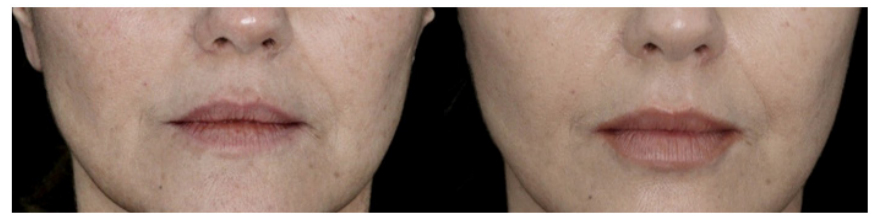

Figure I 4 Before and after treatment based on the Lip Assessment Scale of a 50 -year-old patient.

This patient (Figure 15\&16) presented with the desire of improving her lips. When addressing the entire perioral area, we noticed the volume and proportion of her lips were all in good standing and what she needed the most was more definition and contour. Therefore, we used $0,6 \mathrm{ml}$ of hyaluronic acid only for contouring her lips with a needle and we achieved a satisfying result for both patient and injector.

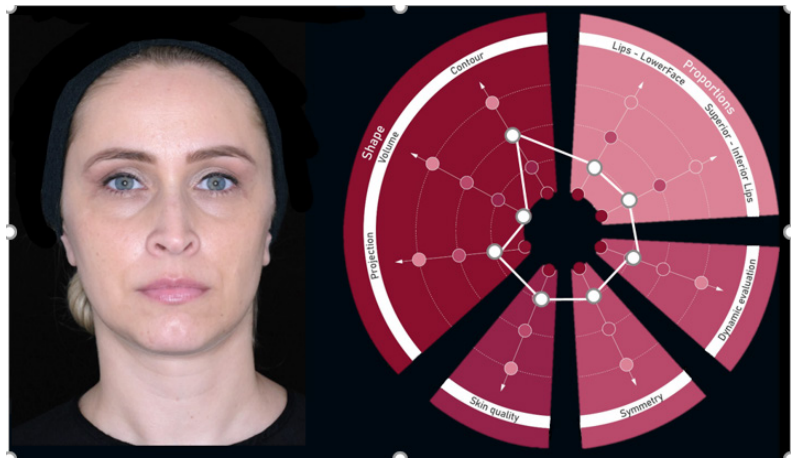

Figure I 5 Example of the Lip and Perioral Assessment Scale showing contour issues.

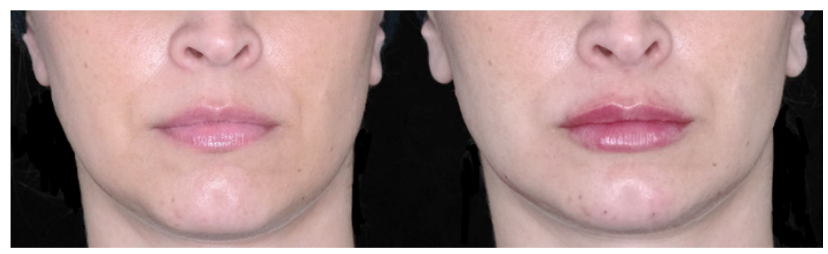

Figure 16 Female, 34 years old - immediate result.

We also see young patients who present with very little problems of symmetry or proportion but that desire more volume to their lips, which can be done but with attention not to change symmetry and proportions that were not an issue to begin with.

\section{Conclusion}

We believe that our Lip and Perioral Assessment Scale is an effective way to systematize the perioral evaluation and help injectors 
identify what the main goals of the treatment should be for each patient and also to identify what should be maintained (as in proportions or projection) even after treating the lips and perioral area.

The scale can be used in any patient that presents with the need of treatment to the lips and perioral area and can improve the time spent with each patient during the assessment and treatment planning. It can also help discussing the treatment options with the patients and delivering a more predictable result, improving overall patient satisfaction.

\section{Conflicts of interest}

The authors declare no conflict of interest.

\section{Acknowledgments}

None.

\section{Funding}

None.

\section{References}

1. Cotofana S, Alfertshofer M, Schenck TL, et al. Anatomy of the Superior and Inferior Labial Arteries Revised: An Ultrasound Investigation and Implication for Lip Volumization. Aesthet Surg J. 2020;40(12):13271335 .

2. Baudoin J, Meuli JN, di Summa PG, et al. A comprehensive guide to upper lip aesthetic rejuvenation. J Cosmet Dermatol. 2019;18(2):444-450.

3. Sahan, Ali \&Tamer, Funda. Four-point injection technique for lip augmentation. Acta Dermatovenerologica Alpina Pannonica et Adriatica. 2018;27.
4. Popenko NA, Tripathi PB, Devcic Z, et al. A Quantitative Approach to Determining the Ideal Female Lip Aesthetic and Its Effect on Facial Attractiveness. JAMA Facial Plastic Surgery. 2017;19(4):261-267.

5. Sarnoff DS, Gotkin RH. Six steps to the "perfect" lip. J Drugs Dermatol. 2012;11(9):1081-1088.

6. Chiu A, Fabi S, Dayan S, Nogueira A. Lip Injection Techniques Using Small-Particle Hyaluronic Acid Dermal Filler. J Drugs Dermatol. 2016;15(9):1076-1082.

7. Trévidic P, Criollo-Lamilla G. French Kiss Technique: An Anatomical Study and Description of a New Method for Safe Lip Eversion. Dermatol Surg. 46(11):1410-1417.

8. Catherine S Chang, Brian L Chang, Michael Lanni, et al. Perioral Rejuvenation: A Prospective, Quantitative Dynamic Three-Dimensional Analysis of a Dual Modality Treatment, Aesthetic Surgery Journal. 2018;38(11):1225-1236.

9. Cotofana S, Pretterklieber B, Lucius R, et al. Distribution Pattern of the Superior and Inferior Labial Arteries: Impact for Safe Upper and Lower Lip Augmentation Procedures. Plast Reconstr Surg. 2017;139(5):10751082.

10. Samizadeh S, Pirayesh A, Bertossi D. Anatomical Variations in the Course of Labial Arteries: A Literature Review. Aesthet Surg J. 2019;39(11):12251235 .

11. Hotta TA. Understanding the Perioral Anatomy. Plast Surg Nurs. 2016;36(1):12-8.

12. Tansatit T, Apinuntrum P, Phetudom T. Cadaveric Assessment of Lip Injections: Locating the Serious Threats. Aesthetic Plast Surg. 2017;41(2):430-440.

13. Ghannam S, Sattler S, Frank K, et al. Treating the Lips and Its Anatomical Correlate in Respect to Vascular Compromise. Facial Plast Surg. 2019;35(2):193-203. 